\title{
New approach for optimizing energy by adjusting the trade-off coefficient in wind turbines
}

\author{
Alireza Fakharzadeh $\mathrm{J}^{1 *}$, Fatemeh Jamshidi ${ }^{2}$ and Leila Talebnezhad ${ }^{1}$
}

\begin{abstract}
Background: As fossil fuels run out, more attention should be paid to renewable energies, among which wind energy is one of the best. Therefore, the optimization of its energetic efficiency in variable speed wind turbines is an important focus of this recent study.

Methods: Based upon linearization, a trade-off between energy conversion maximization and minimization of damage caused by mechanical fatigue, the resulting energy produced by a wind turbine, is optimized. Mathematically, the objective is defined as a stochastic criterion, belonging to the class of linear quadratic regulator (LQR) optimal control problems.

Results: A linear control law has been obtained using an LQR stochastic approach, and the optimal value for a has been calculated using a real-value genetic algorithm. The numerical simulations show a better efficiency for this method compared to other methods used thus far. They also present a better stability when the optimal trade-off coefficient is applied.

Conclusions: The results demonstrate that the curves of the state variables and output variables for the different valves of a converge to zero, which shows that the design controller was fully able to reduce the effectiveness of the white noise.
\end{abstract}

Keywords: Linearization; Linear quadratic regulator; Genetic algorithm; Optimal control; Wind energy

\section{Background}

Renewable energies are obtained from natural resources such as sunlight, wind, rain, tides, and geothermal heat. As fossil fuels become scarce, more attention should be paid to new energy sources or technically new energies. Among the renewable energies, wind energy is known to provide one of the most economical ways to produce electricity because it is inexhaustible and causes no environmental pollution. Moreover, wind turbines normally do not need any extra fuel, water, and other intermediary. Therefore, the exploitation of wind energy using wind turbines for producing electricity has been taken into consideration.

On the other hand, variable-speed fixed-pitch wind turbines are well suited for small- to medium-scale wind power markets due to their simple structure, low cost, and high reliability [1]. Therefore, the optimization of energy efficiency in variable speed for wind turbines is

\footnotetext{
* Correspondence: a_fakharzadeh@sutech.ac.ir

'Department of Mathematics, Shiraz University of Technology, Shiraz 71557-13876, Iran

Full list of author information is available at the end of the article
}

the focus of studies about the designing and exploitation of wind turbines. Indeed, the exclusive goal of wind energy conversion systems is the optimization of the energy conversion with the aim of maximizing the energy captured from wind.

One of the previous studies used variable speed of electrical generators in conjunction with a nonlinear control algorithm (see [2]). Also, Wood (in 2004) employed differential evolution to optimize wind turbine blades (see [3]). Liu et al. (in 2007) presented an optimization model for rotor blades of horizontal axis wind turbines. Their model refers to the wind speed distribution function (see [4]). In another study, a power optimization objective is gained by computing the optimal control settings of wind turbines using data mining and an evolutionary strategy algorithm (see [5]). In this study [6], an approach to perform another linearization for determining an optimal control design was applied. A stochastic model of wind turbines which convert wind speed signals into power output signals with appropriate multifractal statistics was

\section{实}


suggested in [7]. Munteanu et al. [8] have presented a review of both the operational methods for the analysis of the stochastic data and the reconstruction of the detailed stochastic evolution equations from the available data.

Among the recent new works in this area, it is preferred to emphasize not only on an optimization-based approach to reduce extreme structural loads during rapid and emergency shutdown [9], but also on predicting the maximum generation capacity to obtain power control [10] and extreme seeking to perform maximum point tracking [11] as well dynamic responses of land-based and floating wind turbines under pitch system faults [12].

Regarding the mentioned studies, in this current research, we have attempted to optimize the energy in wind turbines by means of simplification using linearization in order to achieve the best adjusting coefficient for the trade-off action via the application of the stochastic LQR method.

Supposing that the energy of the moving wind could be fully captured by the turbine rotor, the total power provided to the rotor would be

$$
P_{t}=\frac{1}{2} \rho a V^{3}
$$

where $\rho$ is the air density, $a$ is the section area of turbine rotation, and $V$ is the wind speed. In fact, this power is partially transferred to the turbine's rotor and transformed into mechanical power, which is further transformed into electrical power by means of an electrical generator. In these transformations, the mechanical power, $P_{\mathrm{wt}}$, that is smaller than the total power, $P_{t}$, is obtained from [13] as

$$
P_{\mathrm{wt}}=\frac{1}{2} \rho a V^{3} C_{p}(\lambda)
$$

Where $C_{p}$ denotes the power coefficient, defining the aerodynamic efficiency of the wind turbine rotor. In fact, $C_{p}$ is a function of the tip speed ratio, scalar $\lambda$, which is defined as follows:

$$
\lambda=\frac{\Omega R}{V},
$$

Where $\Omega$ is the rotational speed of the blades and $R$ is the blade length; and in fact, $\lambda$ is the ratio of the linear speed of blades to wind speed.

In order to reduce mechanical fatigue on the wind turbine system, the torque variations should be minimized by controlling the generator torque variation, $\overline{\Delta \Gamma_{G}}(t)$; hence, due to the stochastic behavior of wind, a stochastic control system is provided.

The most common methods that have been applied for solving this optimal problem can be classified as maximum power point tracking (MPPT) approach, based on an on-off controller [14], fuzzy control techniques [15], linear quadratic Gaussian (LQG) approach [16], and sliding mode techniques [17]. In all of the mentioned methods, the main goal is the maximization of energy efficiency.

In this paper, based on the LQR approach, a new optimal control structure is proposed, which optimizes the combined stochastic criterion that includes the identification of the optimal coefficient for adjusting the given trade-off. Thus, the aim of this paper is to optimize the energy produced by the turbine. To achieve this goal, a real-valued genetic algorithm (GA) and Matlab software tools were applied to obtain the best trade-off coefficient for the variable speed, fixed pitch turbine. Moreover, the LQR stochastic approach is used to design an optimal strategy, which could lead to the optimal solution of the dynamical system.

This paper is organized as follows: in the 'Methods' section, the modeling of the control system is explained, the linearization of the wind energy conversion system is presented, the optimization problem formulation is the focus, and the performance index is introduced. Furthermore, the stochastic LQR controller and the optimal control law are presented. In 'Results and discussion' section, some simulation results are summarized. This paper finishes with some closing remarks in the 'Conclusions' section.

\section{Methods}

\section{Modeling of the wind power system}

The wind energy conversion chain is modeled in Figure 1. It consists of three subsystems: (1) the aerodynamic subsystem $\left(S_{1}\right.$ in Figure 1$)$ is composed of the blades and the hub which capture as much power as possible. (2) The mechanical subsystem $\left(S_{3}\right.$ in Figure 1$)$ is composed of the gearbox (drive train). Gears increase the turbine speed required by most of the generators to produce electricity in an efficient manner.(3) The electromechanical subsystem $\left(S_{2}\right.$ in Figure 1$)$ is composed of the generator. The mechanical power of a wind turbine is converted to electrical power by means of the generator.

By regarding the above-mentioned remarks, the aerodynamic subsystem is modeled by the nonlinear wind torque characteristic [18]:

$$
\Gamma_{\mathrm{wt}}=\Gamma_{\mathrm{wt}}(\Omega, V)=\frac{1}{2} \pi \rho R^{2} V^{3} \frac{C_{p}(\lambda)}{\Omega}=k V^{3} \frac{C_{p}(\lambda)}{\Omega}
$$

Where $\Gamma$ is denoted as the torque ( $\Gamma_{\mathrm{wt}}$ is the electrical and $\Gamma_{G}$ is the mechanical torque) and $k=\frac{1}{2} \pi \rho R^{2}$.

The electromechanical subsystem interacts with the turbine rotor through the drive train; the dynamics of the latter is expressed by

$$
J_{t} \frac{d \Omega}{d t}=\Gamma_{\mathrm{wt}}-\Gamma_{G} .
$$

Where $J_{t}$ expresses the total inertia of the turbine. 


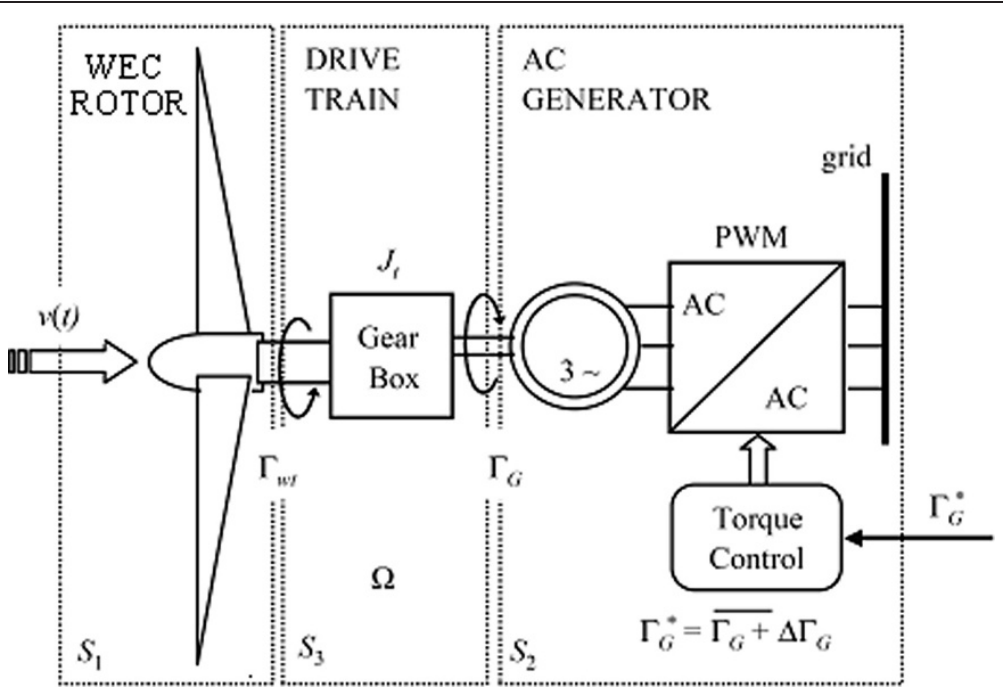

Figure 1 The wind turbine model [16].

The wind model is added to the general model of the system. The wind can be modeled as a stochastic process with two components: the seasonal $\bar{V}$ and the turbulence $\Delta V(t)$ with

$$
V(t)=\bar{V}+\Delta V(t) .
$$

The antagonistic demands of maximizing the energy conversion and minimizing the torque variations are expressed by a combined optimization criterion as [16]

$$
\operatorname{Min}: I=\lim _{t \rightarrow \infty} E\left\{\alpha\left(\lambda(t)-\lambda_{\mathrm{opt}}\right)^{2}\right\}+\lim _{t \rightarrow \infty} E\left\{\overline{\Delta \Gamma}_{G}(t)\right\} .
$$

Here, the positive coefficient $\alpha$ is introduced in the model to adjust the trade-off between the two above-mentioned contrary requirements. We also would like to mention that the scalar $\lambda_{\text {opt }}$ will be introduced in the next section.

\section{Linearization of the system}

Due to the existing difficulties in determining an optimal control for the nonlinear systems, it is necessary to achieve a solution for an equivalent linear control system. In the sequel, the nonlinear relations (4) and (5) will be linearized around the operating point, characterized by a welldefined value of the tip speed ratio, i.e., $\bar{\lambda} \equiv \lambda_{\text {opt }}$. For a variable $x$ of the model, the following notations are adopted:

$$
\bar{x}=\left.x\right|_{\text {optimal operating point }}, \Delta x=x-\bar{x}, \overline{\Delta x}=\frac{\Delta x}{\bar{x}}
$$

Using the Taylor expansion of $\Gamma_{w t}$, Equation 4 is linearized as follows:

$$
\left.\left.\Gamma_{\mathrm{wt}}\right|_{(\Omega, V)} \cong \Gamma_{\mathrm{wt}}\right|_{(\bar{\Omega}, \bar{V})}+\left.\frac{\partial \Gamma_{\mathrm{wt}}}{\partial V}\right|_{(\bar{\Omega}, \bar{V})} \Delta V+\left.\frac{\partial \Gamma_{\mathrm{wt}}}{\partial \Omega}\right|_{(\bar{\Omega}, \bar{V})} \Delta \Omega .
$$

By applying Equations 3 and 4 and assuming $C_{p}^{\prime}(\lambda)=$ $\frac{\partial C_{p}(\lambda)}{\partial \lambda}$ in Equation 9, we obtain

$$
\begin{aligned}
\Gamma_{\mathrm{wt}}=\bar{\Gamma}_{\mathrm{wt}} & +\left(\frac{3 k \bar{V}^{2} C_{p}(\bar{\lambda})}{\bar{\Omega}}-\frac{k \bar{V}^{3} C_{p}^{\prime}(\bar{\lambda}) \bar{\Omega} R}{\bar{\Omega} \bar{V}^{2}}\right) \Delta V \\
& +\left(\frac{k \bar{V}^{3} C_{p}^{\prime}(\bar{\lambda}) \bar{\lambda}}{\bar{\Omega}^{2}}-\frac{k \bar{V}^{3} C_{p}(\bar{\lambda})}{\bar{\Omega}^{2}}\right) \Delta \Omega .
\end{aligned}
$$

Since $\Gamma_{\mathrm{wt}}=\bar{\Gamma}_{\mathrm{wt}}+\Delta \Gamma_{\mathrm{wt}}$, the above equation can be rewritten as

$$
\begin{aligned}
\Delta \Gamma_{\mathrm{wt}}= & \frac{k \bar{V}^{3}}{\bar{\Omega}}\left(3 C_{p}(\bar{\lambda})-C_{p}^{\prime}(\lambda) \bar{\lambda}\right) \overline{\Delta V} \\
& +\frac{k \bar{V}^{3}}{\bar{\Omega}}\left(C_{p}^{\prime}(\bar{\lambda}) \bar{\lambda}-C_{p}(\bar{\lambda})\right) \overline{\Delta \Omega}
\end{aligned}
$$

From Equation 4 and dividing Equation 10 by $\bar{\Gamma}_{\mathrm{wt}}$, the following relation is obtained for $\overline{\Delta \Gamma}_{\mathrm{wt}}$ :

$$
\overline{\Delta \Gamma}_{\mathrm{wt}}=(2-\gamma) \overline{\Delta V}+\gamma \overline{\Delta \Omega}
$$

where $\gamma \equiv \gamma(\bar{\lambda})=\frac{C_{p}^{\prime}(\bar{\lambda}) \bar{\lambda}-C_{p}(\bar{\lambda})}{C_{p}(\bar{\lambda})}$ and is called the torque parameter.

On the other hand, by regarding the definition of the differential, Equation 5 can be approximated as $J_{t} \frac{\Delta \Omega}{\Delta t}=$ $\Gamma_{\mathrm{wt}}-\Gamma_{G}$. Also, by differentiating this relation in respect to time, one can reach

$$
J_{t} \Delta \dot{\Omega}=\overline{\Delta \Gamma}_{\mathrm{wt}} \bar{\Gamma}_{\mathrm{wt}}-\overline{\Delta \Gamma}_{G} \bar{\Gamma}_{G}
$$

Where $\overline{\Delta \Gamma} w t=\frac{\Delta \Gamma_{\mathrm{wt}}}{\bar{\Gamma}_{\mathrm{wt}}}$ and $\overline{\Delta \Gamma} G=\frac{\Delta \Gamma_{G}}{\bar{\Gamma}_{G}}$. Substituting $\Delta \dot{\Omega}=$ $\bar{\Omega} \times \bar{\Omega}$. into Equation 12 and using $\bar{\Gamma}_{\mathrm{wt}}=\bar{\Gamma}_{G}$ gives 
$J_{t} \dot{\bar{\Delta}} \times \bar{\Omega}=\left(\overline{\Delta \Gamma}_{\mathrm{wt}}-\overline{\Delta \Gamma}_{G}\right) \bar{\Gamma}_{\mathrm{wt}}$. Thus, Equation 5 can be represented by the following linearization:

$$
J_{T} \dot{\bar{\Delta}}=\left(\overline{\Delta \Gamma}_{\mathrm{wt}}-\overline{\Delta \Gamma}_{G}\right)
$$

where $J_{T}=\frac{J_{t} \bar{\Omega}}{\bar{\Gamma}_{\mathrm{wt}}}$ is the mechanical time constant.

\section{Optimal control structure}

The linearized relations (11) and (13) are used to represent the state space matrix equations.

Considering $e(t)$ as a white noise as well as $T_{w}=\frac{L}{V}$, where matrix $L$ is the turbulence length, the stochastic model of wind can be presented as (see [16])

$$
\dot{\Delta \vec{V}}(t)=-\frac{1}{T_{w}} \overline{\Delta V}(t)+\frac{1}{T_{w}} e(t)
$$

On the other hand, differentiating Equation 11 with respect to time ( $\gamma$ is a constant) gives

$$
\dot{\bar{\Delta}}_{\mathrm{wt}}(t)=\gamma(\dot{\overline{\Delta \Omega}}(t))+(2-\gamma) \dot{\overline{\Delta V}}(t) .
$$

Now, by substituting Equations 13 and 14 into Equation 15, Equation 16 is obtained:

$$
\dot{\bar{\Delta}}_{\mathrm{wt}}(t)=\frac{\gamma}{J_{T}}\left(\overline{\Delta \Gamma}_{\mathrm{wt}}(t)-\overline{\Delta \Gamma}_{G}(t)\right)+\frac{2-\gamma}{T_{w}}(e(t)-\overline{\Delta V}(t)) .
$$

Hence, Equation 16 together with Equation 13 defines the state space equation as

$$
\dot{X}(t)=A X(t)+B u(t)+L e(t)
$$

where $X(t)=\left[\begin{array}{ll}\overline{\Delta \Omega}(t) & \overline{\Delta \Gamma}_{\text {wt }}(t)\end{array}\right]^{T}$ is the state vector, $u$ $(t)=\overline{\Delta \Gamma}_{G}$ is the control input, and by regarding
Equations 11, 13, and 16, we achieve the following coefficient matrixes:

$$
A=\left[\begin{array}{cc}
0 & \frac{1}{J_{T}} \\
\frac{\gamma}{T_{w}} & \frac{\gamma}{J_{T}}-\frac{1}{T_{w}}
\end{array}\right], B=\left[\begin{array}{c}
-\frac{1}{J_{T}} \\
-\frac{\gamma}{J_{T}}
\end{array}\right], L=\left[\begin{array}{c}
0 \\
\frac{2-\gamma}{T_{w}}
\end{array}\right] .
$$

Moreover, the output variable is defined as being the normalized variation of the tip speed ratio, i.e., $y(t)=\overline{\Delta \lambda}$ $(t)=\frac{\Delta \lambda}{\bar{\lambda}}$. Hence, the output equation is

$$
y(t)=C x(t)
$$

and by considering the principle of superposition, we obtain $C=\left[\begin{array}{cc}\frac{2}{2-\gamma} & \frac{-1}{2-\gamma}\end{array}\right]$. Therefore, relations (17) and (18) represent the linearized model of the wind power system. In this manner, the performance index (7) can be expressed as a quadratic form of the state variables and the control input as follows:

$$
M \text { in }: I=\lim _{t \rightarrow \infty} E\left\{x^{T}(t) Q x(t)+u^{T}(t) u(t)\right\}
$$

where $C_{\alpha}=\sqrt{\alpha} C$ and $Q=C_{\alpha}^{T} C_{\alpha}$ is a positive semidefinite matrix. Hence, the optimal control problem for optimizing the produced energy in the wind turbine can be expressed as

$$
\begin{aligned}
& \text { Min :I }=\lim _{t \rightarrow \infty} E\left\{x^{T}(t) Q x(t)+u^{T}(t) u(t)\right\} \\
& \text { Subject to }: \dot{x}(t)=A x(t)+B u(t)+L e(t),
\end{aligned}
$$

where the disturbance input $e(t)$ is a white noise random signal with spectral density $S_{e}\left(S_{e}=\sigma_{e}^{2}\right)$.

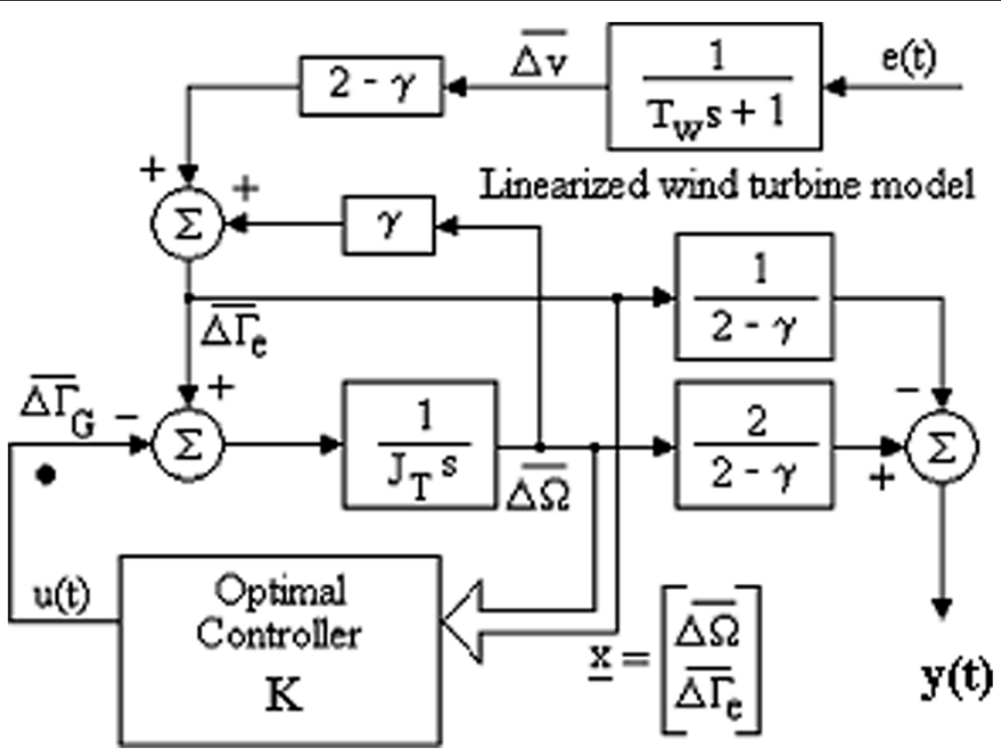

Figure 2 Optimal control scheme [16]. 
As mentioned before, up to now, problem (19) has been solved by different methods such as LQG, MPPT, sliding mode, and fuzzy techniques. But as a different view, Equation 19 is an LQR stochastic problem. So, we present a new and simple solution method in the next subsection.

\section{Optimal LQR stochastic controller design}

In Equation 19, the state equation is linear, the cost function is quadratic, and it is assumed that all states are measurable; thus, the optimal control problem of wind turbine (Equation 19) can classically be considered as an LQR stochastic problem. Based on [19], the unique optimal control input which minimizes the expressed index in Equation 19 for the dynamic system given by relations (17) and (18) is the full state feedback law:

$$
u=-K x(t)
$$

where the feedback matrix, $K$, is introduced by means of

$$
K=R^{-1} B^{T} P
$$

where $P$ must be satisfied by the following algebraic Riccati equation:

$$
P A+A^{T} P+Q-P B R^{-1} B^{T} P=0 .
$$

By applying the diagonalized form of the Hamiltonian matrix, it is proven that the solution of Equation 22 is evaluated as $P=\psi_{21} \psi_{11}^{-1}$ (see [12]), where the columns of $\left[\begin{array}{ll}\psi_{11} & \psi_{12} \\ \psi_{21} & \psi_{22}\end{array}\right]$ are the eigenvectors of the Hamiltonian matrix. Also, the cost function value could be computed by using singular value decomposition as [20]

$$
I=\operatorname{tr}\left[P L S_{e} L^{T}\right]
$$
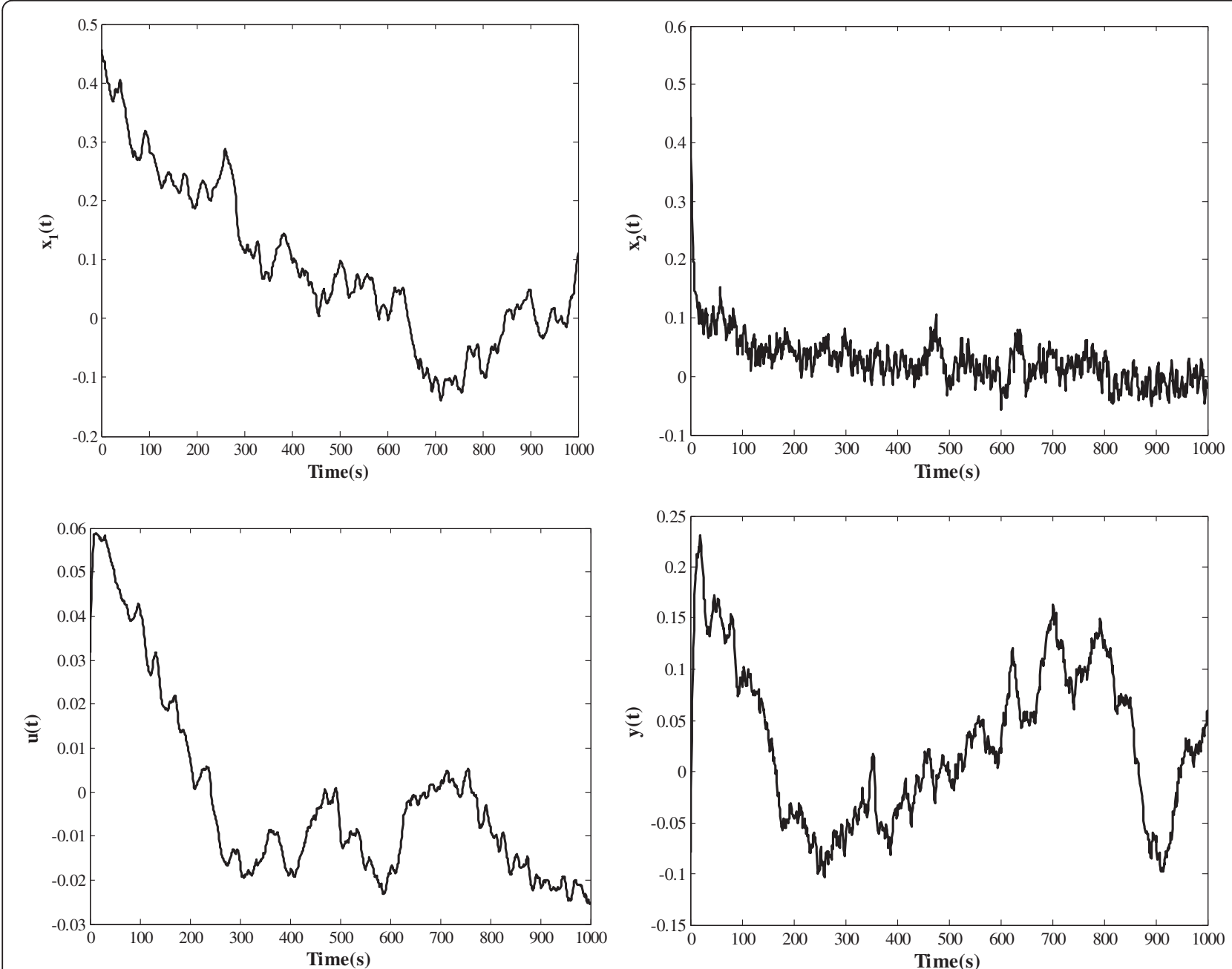

Figure 3 The simulation results of optimal trajectories, output, and control when $a=0.2$ was chosen. 
where the matrix $S_{e}$ is the constant of the white noise. Recalling that the asymptotic stability of the closed-loop system, whose structure is presented in Figure 2 and described by $x \cdot(t)=(A-B K) x(t)$, should be guaranteed. In the next section, the LQR stochastic approach will be used to obtain the optimal control of the given wind turbine system.

\section{Results and discussion}

\section{Simulation results}

The necessary data for performing the simulation are taken from [16], where the proposed control strategy is validated using an electromechanical simulator of the wind energy conversion system. Therefore, the following set of parameters has been used:

$$
\begin{aligned}
J_{t} & =3 \mathrm{~kg} \mathrm{~m}^{2}, R=2.5 \mathrm{~m}, \lambda_{\mathrm{opt}}=7, C_{p \max }=C_{p}\left(\lambda_{\mathrm{opt}}\right) \\
& =0.47 .
\end{aligned}
$$

This provides us with the following values for the linearized system's parameters around the operating point corresponding to $\bar{V}=7 \mathrm{~m} / \mathrm{s}, J_{T}=0.5632, T_{w}=21.4286$ $\mathrm{s}$ and $\gamma=-1$. The standard deviation of $e(t)$ is $\sigma_{e}=0.0475$. The results have shown that the performance index values are sensitive to the $\alpha$ values. So, the real-value genetic algorithm from [21] is used to find the optimal value of $\alpha$.

For this purpose, the crossover relations applied to generate offsprings $\mathrm{ac}_{1}$ and $\mathrm{ac}_{2}$ from parents $a_{1}$ and $a_{2}$, respectively, are as follows:

$$
\begin{aligned}
& \mathrm{rc}=\mathrm{rand}(\text { random variable }) ; \\
& \mathrm{ac}_{1}=\mathrm{rc} \times a_{1}+(1-\mathrm{rc}) \times a_{2} ; \\
& \mathrm{ac}_{2}=(1-\mathrm{rc}) \times a_{1}+\mathrm{rc} \times a_{2} .
\end{aligned}
$$

Also, the following mutation relations used to generate offsprings $\mathrm{am}_{1}$ and $\mathrm{am}_{2}$ from parent $a$ are described as

$$
\begin{aligned}
& r_{f}=\text { rand }, \quad b=3 ; \\
& f=\left(r_{f}\left(1-\frac{\text { itergen }}{\text { gensize }}\right)\right)^{b} ; \\
& \mathrm{am}_{1}=a+(\max (\alpha)-a) \times f ; \\
& \mathrm{am}_{2}=a+(\min (\alpha)+a) \times f,
\end{aligned}
$$
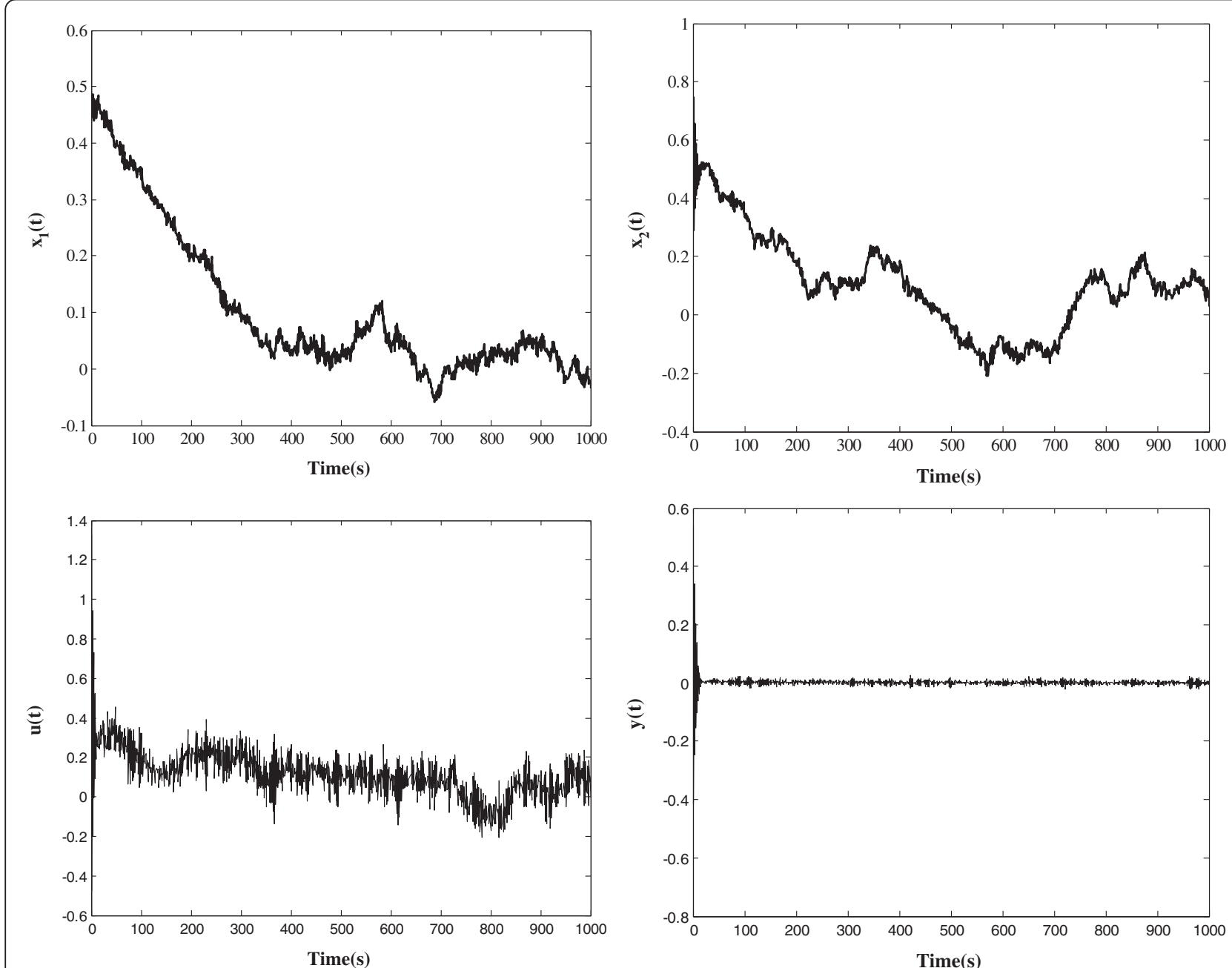

Figure 4 The simulation results of optimal trajectories, output, and control when $a=100$ was chosen. 
where the parameters 'gensize' denotes the population size and 'itergen' denotes the number of iteration of the population (see [6]).

Using the genetic algorithm, the optimal value of $\alpha$ was found to be $\alpha=0.0099$. Moreover, using the Matlab software (R2006A), the gain matrix $K$ and the performance index for several amounts of $\alpha$ are calculated from Equations 21 and 23 as

$$
\begin{aligned}
& K=\left[\begin{array}{ll}
-0.1211 & -0.0257
\end{array}\right], \quad I=3.45 \times 10^{-5} \text { for } \alpha=0.2 \\
& K=\left[\begin{array}{ll}
-6.6399 & 2.4100
\end{array}\right], \quad I=2.28 \times 10^{-4} \text { for } \alpha=100 \\
& K=\left[\begin{array}{ll}
-0.0065 & -0.0016
\end{array}\right], \quad I=2.02 \times 10^{-6} \text { for } \alpha=0.0099 .
\end{aligned}
$$

Obviously, these results show that the best obtained numerical results belong to the optimal value $\alpha=0.0099$, given by applying GA.
Finally, the optimal state vectors, output variable, and control trajectory for these different values of $\alpha$ are illustrated in Figures 3, 4, and 5, respectively.

Comparing these simulation results with those taken from [16] intuitively shows that the corresponding results to $\alpha=0.0099$ do not only have better performance index and stability, but also quite reduced torque variations; this is a good reason for the suitability of $\alpha=0.0099$ when we know that its related eigenvalues of the $A-B K$ matrix are 0.0467 and -1.7843 . It may be necessary to bear in mind that in [6], for a certain case of the objective function, the optimal value of $\alpha$ was obtained as 0.0011 with $I=3 \times 10^{-5}$, but there, the optimal control resulted in an approximated piecewise constant function.

For further research, it is a very interesting and useful idea to measure the noise and find out how much it is reduced. As mentioned in [22] and [23], 'it is a big challenge' and needs some deep experience on concepts like the Langevin and Ornstein-Uhlenbeck process.
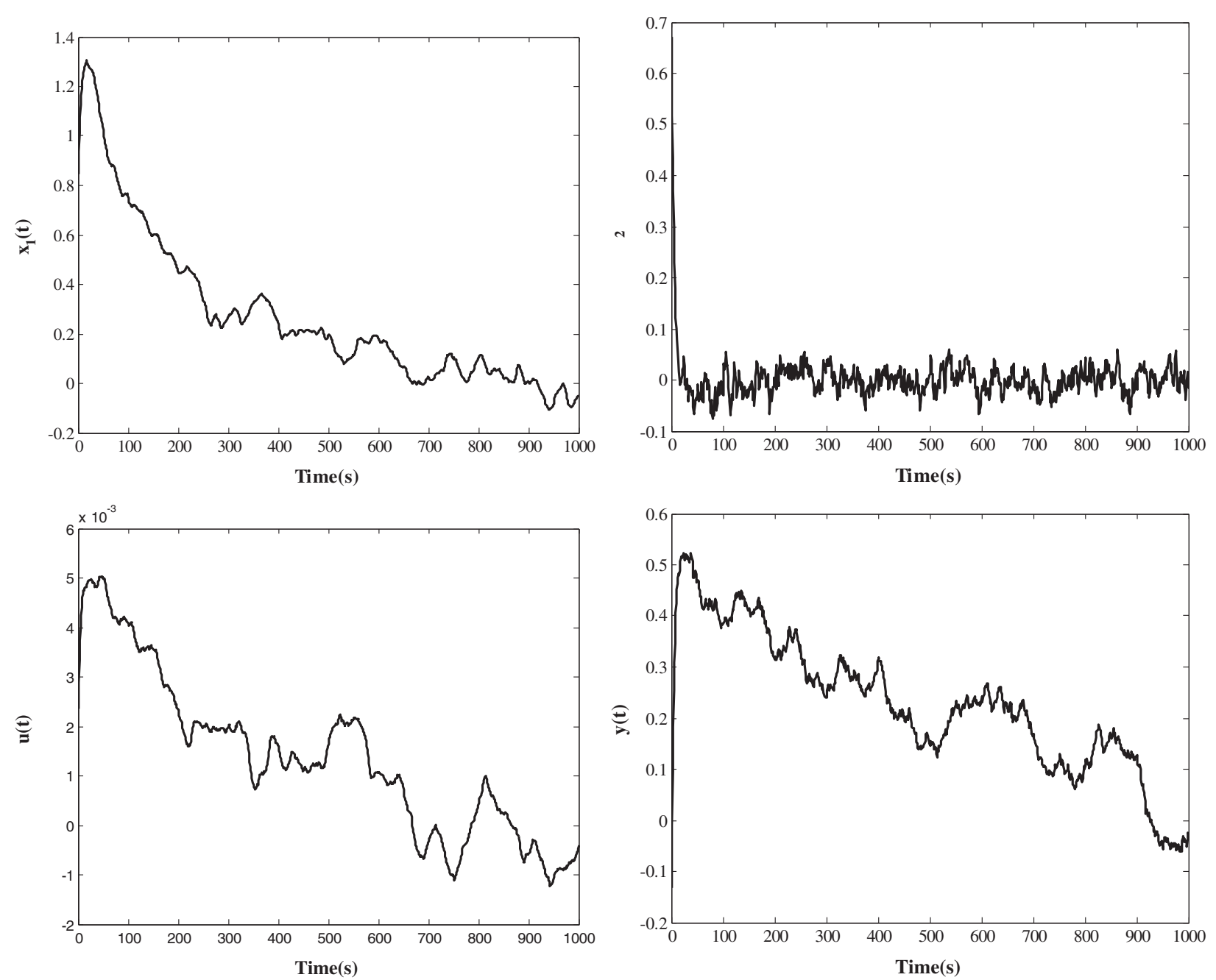

Figure 5 The simulation results for the best $a=0.0099$ obtained from applying GA. 


\section{Conclusions}

This paper proposes an optimal control strategy for variable-speed fixed-pitch wind turbines. The optimality of the whole system is defined in relation to the tradeoff between wind energy conversion maximization and the minimization of the generator torque variation. This optimal problem is treated using an LQR stochastic approach, whose effectiveness was proven by a numerical solution. Since this combination is dependent on the definition parameter for the required trade-off, this method is able to define the parameter in an optimal way by genetic algorithms. Applying the best of the obtained trade-off coefficients in an LQR stochastic approach allows us not only to produce a larger amount of energy, but also to obtain a better stability; moreover, the torque variations were extremely reduced and the numerical conclusion showed the desired ability and application of this method. These results demonstrated that the curves of the state variables and output variables for the different values of $\alpha$ converge to zero, which shows that the design controller was fully able to reduce the effectiveness of the white noise.

\section{Competing interests}

The authors declare that they have no competing interests.

\section{Authors' contributions}

All authors together prepared the manuscript in the field of optimization of energy in wind turbine. The roles of contributors are as follows: AFJ is the adviser professor expert in optimal control, optimal shape design, and optimization. FJ scientifically collaborated with experts in optimal control. LT contributed her expertise in optimization and control. All authors read and approved the final manuscript.

\section{Author details}

${ }^{1}$ Department of Mathematics, Shiraz University of Technology, Shiraz 71557-13876, Iran. ²Department of Electrical Engineering, Fasa University, Fasa 74617-81189, Iran.

Received: 16 August 2012 Accepted: 8 September 2013 Published: 18 September 2013

\section{References}

1. Chen J, Gong C (2013) New overall power control strategy for variablespeed fixed-pitch wind turbines within the whole wind velocity range. IEEE Trans Ind Electron 60(7):2652-2660

2. Costa P, Mrtins A, Carvalho A (2005) Wind energy extraction and conversion: optimization through variable speed generators and nonlinear fuzzy control. IEEE Proceeding-Electric Power Applications 139(5):421-428

3. Wood DH (2004) Dual purpose design of small turbine blades. Wind Eng 28(5):511-527

4. Liu X, Chen Y, Ye Z (2007) Optimization model for rotor blades of horizontal axis wind turbines. Front Mech Eng China 2(4):483-488

5. Kusiak A, Zheng H, Song Z (2009) Power optimization of wind turbines with data mining and evolutionary computation. Renew Energy 35:695-702

6. Fakharzadeh JA, Talebnezhad L (2011) A linear approach for optimizing energy in wind turbines by embedding process. Int J Innovat Comput Inform Contr 7:4465-4476, No.7(B)

7. Milan P, Wächter M, Peinke J (2013) Turbulent character of wind energy. Phys Rev Lett 110(13):137403-137407

8. Friedrich R, Peinkeb J, Sahimi M, RahimiTabar MR (2011) Approaching complexity by stochastic methods: from biological systems to turbulence. Phys Rep 506(5):87-162
9. Gros S, Chachuat B (2013) Optimization-based load reduction during rapid shutdown of multi-megawatt wind turbine generators. Wind Energy J, doi:10.1002/we.1618

10. Liu D, Guo G, Wang W (2013) An active power control strategy for wind frame based on predictions of wind turbine's maximum generation capacity. Renew Sustain Energ 5(013121):1-10

11. Ghaffari A (2013) Power optimization and control in wind energy conversion system using extremum seeking. Research report, Jacobs University

12. Jiang Z, Karimirad M, Moan T (2013) Dynamic response analysis of wind turbines under blade pitch system fault, grid loss, and shutdown events. Wind Energy J, 10.1002/we.1639

13. Burton T, Sharpe D, Jenikins N, Bossanyi E (2001) Wind energy hand book. Wiley, New York

14. Bhowmik S, Spee R (1998) Wind speed estimation based variable speed wind power generation. In: IECON'98. Proceedings of the Annual IEEE Conference of the Industrial Electronics Society, Aachen, 31 Aug-4 Sep 1998 vol 2. IEEE, Piscataway, pp 596-601

15. Simoes MG, Boes BK, Spiegel RJ (1997) Fuzzy logic based intelligent control of a variable speed cage machine wind generation system. IEEE Trans Power Electron 12(1):87-95

16. Munteanu I, Cutuluis NA, Bratcu Al, Ceanga E (2005) Optimization of variable speed wind power systems based on a LQG approach. Control Eng Pract 13:903-912

17. De Battist H, Mantz RJ, Christiansen CF (2000) Dynamical sliding mode power control of wind driven induction generators. IEEE Trans Energ Convers 15(4):451-457

18. Wilkie J, Leithead WE, Anderson C (1990) Modeling of wind turbines by simple models. Wind Eng 4:247-274

19. Sinha A (2007) Linear systems optimal and robust control. CRC, Boca Raton, p 601

20. Jeffrey BB (1999) Linear optimal control $\mathrm{H}_{2}$ and $\mathrm{H}_{\infty}$ methods. AddisonWesley, London, p 601

21. Fogarty TC (1989) Varying the probability of mutation in genetic algorithm In: Proceedings of the Third International Conference on Genetic Algorithms, Fairfax, June 4-7 1989. Morgan Kaufmann, San Francisco, pp 104-109

22. Boettcher F, Peinke J, Kleinhans D, Friedrich R, Lind PG, Haase M (2006) Reconstruction of complex dynamical systems affected by strong measurement noise. Phys Rev Lett 97:090603, 1-4, nlin.CD/0607002

23. Lind PG, Hasse M, Bottcher F, Peinke J, Kleinhanse D, Friedrich R (2012) Extracting strong measurement noise from stochastic series: application to empirical data. Phys Rev E 81(041125):1-13

doi:10.1186/2192-0567-3-19

Cite this article as: Fakharzadeh J et al.: New approach for optimizing energy by adjusting the trade-off coefficient in wind turbines. Energy, Sustainability and Society 2013 3:19.

\section{Submit your manuscript to a SpringerOpen ${ }^{\odot}$ journal and benefit from:}

- Convenient online submission

- Rigorous peer review

- Immediate publication on acceptance

- Open access: articles freely available online

- High visibility within the field

- Retaining the copyright to your article

Submit your next manuscript at $>$ springeropen.com 